\title{
Many-body calculations of plasmon and phonon satellites in angle-resolved photoelectron spectra using the cumulant expansion approach
}

\author{
Fabio Caruso, ${ }^{1}$ Carla Verdi, ${ }^{2}$ and Feliciano Giustino ${ }^{2,3, *}$ \\ ${ }^{1}$ Institut für Physik and IRIS Adlershof, Humboldt-Universität zu Berlin, Berlin, Germany \\ ${ }^{2}$ Department of Materials, University of Oxford, Parks Road, Oxford OX1 3PH, United Kingdom \\ ${ }^{3}$ Department of Materials Science and Engineering, \\ Cornell University, Ithaca, New York, 14850, USA
}

\begin{abstract}
The interaction of electrons with crystal lattice vibrations (phonons) and collective charge-density fluctuations (plasmons) influences profoundly the spectral properties of solids revealed by photoemission spectroscopy experiments. Photoemission satellites, for instance, are a prototypical example of quantum emergent behavior that may result from the strong coupling of electronic states to plasmons and phonons. The existence of these spectral features has been verified over energy scales spanning several orders of magnitude (from $50 \mathrm{meV}$ to $15-20 \mathrm{eV}$ ) and for a broad class of compounds such as simple metals, semiconductors, and highly-doped oxides. During the past few years the cumulant expansion approach, alongside with the $G W$ approximation and the theory of electron-phonon and electron-plasmon coupling in solids, has evolved into a predictive and quantitatively accurate approach for the description of the spectral signatures of electron-boson coupling entirely from first principles, and it has thus become the state-of-the-art theoretical tool for the description of these phenomena. In this chapter we introduce the fundamental concepts needed to interpret plasmon and phonon satellites in photoelectron spectra, and we review recent progress on first-principles calculations of these features using the cumulant expansion method.
\end{abstract}

\section{INTRODUCTION}

The emergence of satellites in photoemission spectroscopy provides direct evidence of the electronic coupling to bosonic excitations in solids. Satellites are spectral features that reflect the simultaneous excitation of a hole and of a boson, and they are separated from the quasiparticle peak by a multiple of the boson energy. The origin of these features may be understood based on simple considerations on the energy scales involved in the photoemission process. When a photon with energy $\hbar \omega$ is absorbed by an electron with binding energy $\epsilon_{i}$, if no boson modes are excited in the system, energy conservation requires the condition $\hbar \omega=\epsilon_{i}+\Phi+E_{\text {kin }}$ to be satisfied, where $\Phi$ is the work-function of the system and $E_{\text {kin }}$ is the kinetic energy of the photo-emitted electron. In photoemission, by measuring $E_{\text {kin }}$ and $\Phi$, the electron binding energy can thus be inferred. If, in addition to the creation of a hole, a fraction of the absorbed photon energy is transferred to the system in the form of bosonic modes, such as plasmons and phonons, the energy conservation condition is modified as follows: $E_{\text {kin }}=\hbar \omega-\epsilon_{i}-n E_{\mathrm{b}}-\Phi$, where $E_{\mathrm{b}}$ is the energy of the boson and $n$ an integer. Since $\hbar \omega$ and $\Phi$ are constants, the kinetic energy distribution of the photo-emitted electrons will be peaked at the energies corresponding to (i) the binding energy of electrons $\epsilon_{i}$ and (ii) the sum of the binding and boson energies $\epsilon_{i}+n E_{\mathrm{b}}$, and it may thus provide direct information regarding the coupling of electrons to bosonic modes in solids.

The presence of satellites in the photoemission spectra of solids was first predicted by a theoretical analysis of

\footnotetext{
* feliciano.giustino@materials.ox.ac.uk
}

the spectral function of the homogeneous electron gas by [1-3], and subsequently verified experimentally for the core electrons of simple metals 4]. Recently, the availability of energy resolutions of the order of $25-50 \mathrm{meV}$ in angle-resolved photoelectron spectroscopy (ARPES) made it possible to observe new low-energy signatures of electron-boson coupling in experiments. In particular, high-resolution ARPES measurements of graphene by [5] have revealed plasmon-induced satellite structures with characteristic energies of the order of $\sim 1 \mathrm{eV}$. More recently, polaronic satellites at energies of the order $\sim 100 \mathrm{meV}$ from the band edges have been observed in doped oxides, for example by [6, 7]. At variance with valence-plasmon satellites, which typically appear at energies between 5 and $15 \mathrm{eV}$ below the Fermi energy and have been known since the early days of photoemission spectroscopy, low-energy satellites are a manifestation of the coupling between low-energy bosonic modes and electronic carriers near the band edges. In addition to the formation of satellites, the coupling to bosons may lead to the emergence of photoemission kinks [8, 9] and to a renormalization of energy levels [10 12 and carrier lifetimes [13, 14.

In this chapter we will discuss the state-of-the-art techniques for the description of plasmon and polaron satellites and their application to the prediction and interpretation of photoemission spectroscopy experiments.

\section{THE LOCALIZED-ELECTRON MODEL}

To illustrate how the interaction between electrons and bosons may lead to the emergence of satellites in photoemission spectra, we consider in the following the exactly solvable model of a "localized electron" in a solid inter- 
acting with a boson bath. The latter can be regarded as a set of phonons, plasmons, or any other bosonic excitations that may be approximately represented as a set of uncoupled harmonic oscillators. The localized electron is assumed to be dispersionless, that is, its energy $\varepsilon$ is independent of the crystal momentum, and its interaction with other electrons in the system is neglected. Instances in which the electron energy levels exhibit a weak dependence on momentum, and can thus be approximated as non-dispersive, are for example core electrons in solids, localized impurity levels, and $4 f$ electrons. On the other hand, electron-electron interactions are typically strong and non-negligible in three-dimensional solids, which poses limitations to the applicability of this model to real physical systems. This simplified model, however, is remarkably successful in describing the emergence of bosonic satellites in the spectral properties, and is in good qualitative agreement with more advanced theories whereby the electron-electron interaction is accounted for. This is demonstrated, for instance, by the generalization of the localized electron model reported by [3].

The localized electron model is described by the following electron-boson coupling Hamiltonian:

$$
\begin{aligned}
\hat{H} & =\hat{H}_{\mathrm{e}}+\hat{H}_{\mathrm{b}}+\hat{H}_{\mathrm{int}} \\
& =\varepsilon \hat{c}^{\dagger} \hat{c}+\sum_{\mathbf{q}} \hbar \omega_{\mathbf{q}} \hat{b}_{\mathbf{q}}^{\dagger} \hat{b}_{\mathbf{q}}+\sum_{\mathbf{q}} g_{\mathbf{q}} \hat{c}^{\dagger} \hat{c}\left(\hat{b}_{\mathbf{q}}+\hat{b}_{-\mathbf{q}}^{\dagger}\right),
\end{aligned}
$$

where $\hat{c}^{\dagger}$ and $\hat{c}$ are fermionic creation and annihilation operators for the localized electron, respectively, which satisfy the ordinary anti-commutation relations. Similarly, the operators $\hat{b}_{\mathbf{q}}^{\dagger}$ and $\hat{b}_{\mathbf{q}}$ respectively create and annihilate a boson with energy $\hbar \omega_{\mathbf{q}}$ and momentum $\mathbf{q}$ and satisfy bosonic commutation relations. The absence of two-particle interaction terms in the Hamiltonian reflects the fact that both electron-electron and boson-boson interactions are neglected. The localized electron interacts with the boson bath with the coupling strength $g_{\mathbf{q}}$.

As we are primarily interested in the effects of the electron-boson interaction on the photoemission intensity, the relevant quantity that we want to compute is the electron spectral function:

$$
A(\omega)=-\frac{1}{\pi} \operatorname{Im} G^{\mathrm{ret}}(\omega)
$$

with the single-particle retarded Green's function $G^{\text {ret }}$ defined as:

$$
G^{\mathrm{ret}}(t)=-i\left\langle\Psi_{0}\left|\left\{\hat{c}(t), \hat{c}^{\dagger}(0)\right\}\right| \Psi_{0}\right\rangle \theta(t),
$$

where $\{$,$\} denotes the anticommutator, \Psi_{0}$ the electronic ground state, and the time-dependence of the operators is accounted for in the Heisenberg picture. As shown by [3], the Green's function associated to the Hamiltonian in Eq. (1) can be calculated exactly. In fact, by applying a unitary transformation, Eq. (1) is recast in the form of a shifted harmonic oscillator Hamiltonian for which eigenvalues and eigenvectors are known [15]. The spectral function for a localized electron can thus be expressed as [3]:

$$
A(\omega)=\sum_{n=0}^{\infty} \frac{e^{-a} a^{n}}{n !} \delta\left(\hbar \omega-\varepsilon-a \hbar \omega_{\mathrm{b}}+n \hbar \omega_{\mathrm{b}}\right),
$$

where $a=\sum_{\mathbf{q}} g_{\mathbf{q}}^{2} /\left(\hbar \omega_{\mathrm{b}}\right)^{2}$ and for simplicity the energy of the boson mode has been replaced by its average value $\hbar \omega_{\mathrm{b}}$.

In the small coupling limit, that is for $g_{\mathbf{q}} \rightarrow 0$, the spectral function reduces to the case of a non-interacting electron $A(\omega)=\delta(\hbar \omega-\varepsilon)$, and the Dirac delta function is peaked at the quasiparticle energy. For finite coupling strengths, the structure of the spectral function in Eq. (4) reveals that the effect of the interaction between electrons and bosons on the spectral properties of the system is twofold. First, the quasiparticle energy of the localized electron is shifted by $a \hbar \omega_{\mathrm{b}}$. This process is analogous, for example, to the well-known band-gap renormalization of semiconductors and insulators due to the electron-phonon interaction [16, 17] and it results from the dressing of the bare quasiparticle via the interaction with the boson modes. Second, the spectral function exhibits a series of additional features at lower energies which are separated from the quasiparticle peak by multiples of the boson energy $\hbar \omega_{\mathrm{b}}$. These spectral features arise from the simultaneous excitation of the localized electron and of one or more bosons with energy $\hbar \omega_{\mathrm{b}}$.

In Fig. 1(a) we show the spectral function obtained from Eq. (4) considering $\varepsilon=-40 \mathrm{meV}, \hbar \omega_{\mathrm{b}}=100 \mathrm{meV}$, $g_{\mathbf{q}}=100 \mathrm{meV}$. A picture in closer agreement with angle-resolved photoemission spectroscopy is obtained when considering the case of dispersive electronic states: Fig. 1(b) illustrates the spectral intensity map for a parabolic band, obtained by replacing the electronic energy with $\varepsilon_{\mathbf{k}}=\varepsilon+\hbar^{2} k^{2} / 2 m$ in Eq. (4). This simple generalization of the localized electron model illustrates that, in presence of non-trivial energy-wavevector dispersion relations, the energy of the satellite features induced by electron-boson coupling also acquires a dependence on the crystal momentum that follows closely the dispersion of the ordinary quasiparticle states. This phenomenon translates into the formation of plasmonic polaron bands due to electron-plasmon coupling [18-22 and polaron satellites due to electron-phonon coupling [6, 7, 23] in the ARPES spectra of semiconductors and $n$-doped oxides, respectively.

Overall, the solution of the localized-electron model reveals that the spectral function of a system of interacting electrons and bosons, whereby the interaction is described by the last term of Eq. (1), may exhibit a series of satellite structures, with binding energy blueshifted with respect to the main quasiparticle peak by multiples of the boson energy. Despite the simplicity of the model, this result provides a first indication that the coupling to plasmons and phonons in real systems, in which the coupling Hamiltonian assumes a similar form, may also 


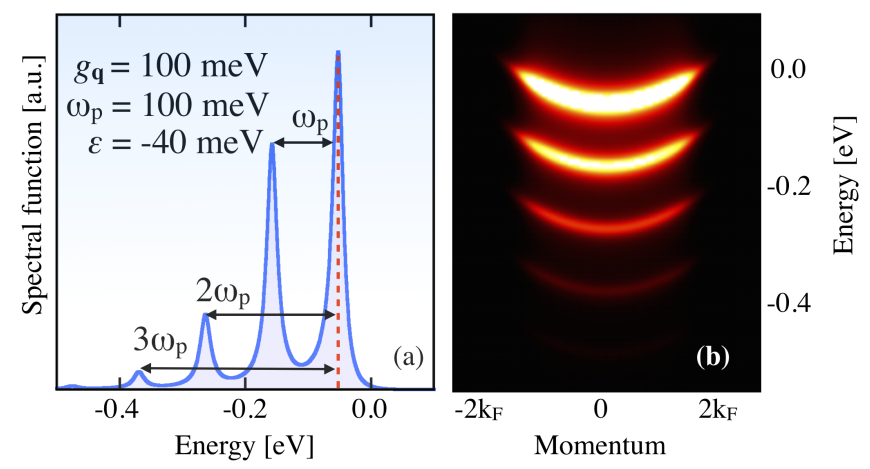

FIG. 1. (a) Spectral function of the localized electron model evaluated using Eq. (4) for a non-dispersive electron with binding energy $\varepsilon$ coupled to a boson with frequency $\omega_{\mathrm{b}}$ with a coupling strength $g_{\mathbf{q}}$. (b) Spectral intensity map for an electron with parabolic band dispersion $\varepsilon_{\mathbf{k}}=\varepsilon+\hbar^{2} k^{2} / 2 m$. The Dirac $\delta$ functions in Eq. (4) have been replaced by Lorentzian functions with a $20 \mathrm{meV}$ broadening.

induce the formation of satellite features for sufficiently strong coupling.

\section{FIRST-PRINCIPLES DESCRIPTION OF SATELLITES IN PHOTOEMISSION}

Despite the different nature of plasmon and phonon collective excitations in solids, the many-body theory of electron-boson interaction represents the common playground to describe their coupling to electronic states, and to investigate the spectral fingerprints resulting from this interaction. The Hedin-Baym equations [17 based on many-body perturbation theory (MBPT) provide a formally exact framework to investigate the coupling to plasmons and phonons, and are the starting point for the theoretical description of satellites in photoemission spectra. The electron self-energy for the coupled electronphonon system in the Migdal approximation, that is neglecting vertex corrections, is given by [2, 17]:

$\Sigma(\mathbf{k}, \omega)=i \int \frac{d \mathbf{q}}{\tilde{\Omega}} \frac{d \omega}{2 \pi} G\left(\mathbf{k}+\mathbf{q}, \omega+\omega^{\prime}\right)\left[W_{\mathrm{e}}\left(\mathbf{q}, \omega^{\prime}\right)+W_{\mathrm{ph}}\left(\mathbf{q}, \omega^{\prime}\right)\right]$

where $\tilde{\Omega}$ is the reciprocal-space volume, $G$ is the single-particle Green's function and $W_{\mathrm{e}}\left(W_{\mathrm{ph}}\right)$ is the screened Coulomb interaction due to the electronelectron (electron-phonon) interaction. It can be shown that the electron-phonon part may be expressed as $W_{\mathrm{ph}}=W_{\mathrm{e}} D W_{\mathrm{e}}$ (in symbolic notation), where $D$ is the density-density correlation function for the nuclear fluctuations. Eq. (5) neglects the so-called Debye-Waller contribution to the self-energy, however this contribution is frequency-independent and therefore does not give rise to additional structure in the electron spectral function [17.

If the nuclei are treated in the clamped-ion approximation, that is $W_{\mathrm{ph}}(\mathbf{q}, \omega)=0$, the ordinary $G W$ approximation is recovered. By expanding the Bloch wavefunctions in a basis set of plane waves, $\psi_{n \mathbf{k}}(\mathbf{r})=$ $\sum_{\mathbf{G}} c_{n \mathbf{k}}(\mathbf{G}) e^{i(\mathbf{k}+\mathbf{G}) \cdot \mathbf{r}}$, the $G W$ self-energy $\Sigma^{G W}$ can be expressed in a form more suitable for first-principles calculations of crystalline solids [24:

$$
\begin{aligned}
\Sigma_{n \mathbf{k}}^{G W}(\omega)=\frac{i \hbar}{2 \pi} \sum_{m \mathbf{G G}^{\prime}} & \int \frac{d \mathbf{q}}{\Omega_{\mathrm{BZ}}} M_{\mathbf{G}}^{m n}(\mathbf{k}, \mathbf{q})^{*} M_{\mathbf{G}^{\prime}}^{m n}(\mathbf{k}, \mathbf{q}) \\
& \times \int d \omega^{\prime} \frac{v_{\mathbf{G}}(\mathbf{q}) \epsilon_{\mathbf{G}, \mathbf{G}^{\prime}}^{-1}(\mathbf{q}, \omega)}{\hbar \omega+\hbar \omega^{\prime}-\tilde{\varepsilon}_{m \mathbf{k}+\mathbf{q}}}
\end{aligned}
$$

where $M_{\mathbf{G}}^{m n}(\mathbf{k}, \mathbf{q})=\left\langle\psi_{m \mathbf{k}+\mathbf{q}}\left|e^{i(\mathbf{q}+\mathbf{G}) \cdot \mathbf{r}}\right| \psi_{n \mathbf{k}}\right\rangle$ are the optical matrix elements, $\Omega_{\mathrm{BZ}}$ is the volume of the Brillouin zone, and $v_{\mathbf{G}}(\mathbf{q})=e^{2} / \varepsilon_{0}|\mathbf{q}+\mathbf{G}|^{2}$ ( $\varepsilon_{0}$ is the vacuum permittivity). We defined $\tilde{\varepsilon}_{m \mathbf{k}+\mathbf{q}}=\varepsilon_{m \mathbf{k}+\mathbf{q}}-$ $i \eta \operatorname{sgn}\left(\varepsilon_{m \mathbf{k}+\mathbf{q}}\right)$, with $\eta$ a positive infinitesimal and $\varepsilon_{m \mathbf{k}+\mathbf{q}}$ the Bloch electron energy relative to the chemical potential $\mu$. The dielectric matrix $\epsilon_{\mathbf{G}, \mathbf{G}^{\prime}}(\mathbf{q}, \omega)$ is related to the screened Coulomb interaction via $W_{\mathbf{G}, \mathbf{G}^{\prime}}(\mathbf{q}, \omega)=$ $v_{\mathbf{G}}(\mathbf{q}) \epsilon_{\mathbf{G}^{\prime}, \mathbf{G}^{\prime}}^{-1}(\mathbf{q}, \omega)$. In $G W$ calculations, the dielectric function is typically expressed as $\epsilon_{\mathbf{G}, \mathbf{G}^{\prime}}(\mathbf{q}, \omega)=\delta_{\mathbf{G}, \mathbf{G}^{\prime}}-$ $v_{\mathbf{G}}(\mathbf{q}) \chi_{\mathbf{G}, \mathbf{G}^{\prime}}^{0}(\mathbf{q}, \omega)$, where $\chi_{\mathbf{G}, \mathbf{G}^{\prime}}^{0}$ is the independentparticle polarizability (see, e.g., 24]).

The second term in Eq. (5) represents the electronphonon self-energy $\Sigma^{\mathrm{ep}}$ in the Migdal approximation. Its expression in the basis of single-particle Bloch wavefunctions reads:

$$
\begin{aligned}
& \Sigma_{n \mathbf{k}}^{\mathrm{ep}}(\omega)=\sum_{m \nu} \int \frac{d \mathbf{q}}{\Omega_{\mathrm{BZ}}}\left|g_{m n \nu}(\mathbf{k}, \mathbf{q})\right|^{2} \times \\
& {\left[\frac{n_{\mathbf{q} \nu}+f_{m \mathbf{k}+\mathbf{q}}}{\hbar \omega-\varepsilon_{m \mathbf{k}+\mathbf{q}}+\hbar \omega_{\mathbf{q} \nu}+i \eta}+\frac{n_{\mathbf{q} \nu}+1-f_{m \mathbf{k}+\mathbf{q}}}{\hbar \omega-\varepsilon_{m \mathbf{k}+\mathbf{q}}-\hbar \omega_{\mathbf{q} \nu}+i \eta}\right],}
\end{aligned}
$$

where $n_{\mathbf{q} \nu}$ and $f_{m \mathbf{k}+\mathbf{q}}$ are the Bose-Einstein and FermiDirac distributions, respectively. Eq. (7) is derived after transforming the frequency integration in Eq. (5) into a Matsubara summation to extend the formalism to finite temperatures, and performing the integration analytically by using the expressions for the unperturbed electron and phonon Green's functions. The self-energy is then analytically continued to the real frequency axis [15, and only the diagonal terms are retained, as in Eq. (6). The electron-phonon matrix element $g$ is defined as:

$$
g_{m n \nu}(\mathbf{k}, \mathbf{q})=\left\langle\psi_{m \mathbf{k}+\mathbf{q}}\left|\Delta_{\mathbf{q} \nu} V_{\mathrm{KS}}\right| \psi_{n \mathbf{k}}\right\rangle .
$$

and it contains the variation of the self-consistent KohnSham (KS) 25] potential $V_{\mathrm{KS}}$ with respect to a phonon perturbation. The umklapp processes are included by letting $\mathbf{k}+\mathbf{q}$ fall outside the first Brillouin zone and folding it back with a reciprocal lattice vector $\mathbf{G}$. The definition in Eq. (8) corresponds to taking the bare Coulomb potential between the electrons and the nuclei screened by the electronic dielectric function $\epsilon_{\mathbf{G}, \mathbf{G}^{\prime}}(\mathbf{q}, \omega)$. In principle 
the matrix element should be frequency dependent, however in ab initio calculations it is taken to be static, following the adiabatic approximation of standard densityfunctional theory (DFT). In Sec. V] we will discuss how going beyond this approximation is needed when describing polarons in ARPES spectra. In practical calculations Eq. (8) is evaluated using density-functional perturbation theory (DFPT) by determining the linear variation of the self-consistent Kohn-Sham potential. A rigorous discussion of the calculation of the DFPT screening as compared to the many-body random-phase approximation (RPA) can be found for example in [26].

\section{A. The electron spectral function}

The calculation of the self-energies defined by Eqs. (6) and (7) constitutes the first step towards the description of satellites from first principles. Details regarding the numerical evaluation of these expressions have been thoroughly reported for instance in [27] and [28] and will not be discussed here. Once the electron selfenergy $\Sigma_{n \mathbf{k}}(\omega)$ is known, the spectral function is obtained by combining Eq. (2) with the Dyson's equation $G_{n \mathbf{k}}=\left[\hbar \omega-\varepsilon_{n \mathbf{k}}-\Sigma_{n \mathbf{k}}(\omega)\right]^{-1}$, which yields:

$$
A(\mathbf{k}, \omega)=-\frac{1}{\pi} \sum_{n} \frac{\operatorname{Im} \Sigma_{n \mathbf{k}}(\omega)}{\left[\hbar \omega-\varepsilon_{n \mathbf{k}}-\operatorname{Re} \Sigma_{n \mathbf{k}}(\omega)\right]^{2}+\left[\operatorname{Im} \Sigma_{n \mathbf{k}}(\omega)\right]^{2}}
$$

The spectral function exhibits sharp peaks whenever the first term in the denominator of Eq. (9] $\left[\hbar \omega-\varepsilon_{n \mathbf{k}}-\right.$ $\operatorname{Re} \Sigma_{n \mathbf{k}}(\omega)$ ] vanishes or has a minimum. In particular, quasiparticle peaks in the spectral function arise at the energies $\hbar \omega=\varepsilon_{n \mathbf{k}}+Z_{n \mathbf{k}} \operatorname{Re} \Sigma_{n \mathbf{k}}\left(\varepsilon_{n \mathbf{k}}\right)$, where $Z_{n \mathbf{k}}=[1-$ $\left.\partial \operatorname{Re} \Sigma_{n \mathbf{k}}(\omega) /\left.\partial \omega\right|_{\omega=\varepsilon_{n \mathbf{k}}}\right]^{-1}$ is the quasiparticle weight. If the Bloch single-particle energies $\varepsilon_{n \mathbf{k}}$ are obtained from a DFT calculation, Eq. (9) should be modified to avoid double counting of the exchange-correlation [24].

A more suitable framework for the evaluation of satellites in photoemission is provided by the cumulant expansion approach. The cumulant expansion is an alternative formulation of the (retarded) single-particle Green's function which is in principle exact. The Green's function is expressed in the form [21, 29]:

$$
G_{n \mathbf{k}}(t)=i \theta(t) \exp \left[-i\left(\varepsilon_{n \mathbf{k}}-i \eta\right) t / \hbar+C_{n \mathbf{k}}(t)\right]
$$

where we introduced the cumulant function $C_{n \mathbf{k}}(t)$ which is defined by:

$$
C_{n \mathbf{k}}(t)=\frac{1}{\hbar \pi} \int d \omega \operatorname{Im} \Sigma_{n \mathbf{k}}\left(\varepsilon_{n \mathbf{k}} / \hbar-\omega\right) \frac{1-e^{i \omega t}+i \omega t}{\omega^{2}}
$$

In practice, the spectral function obtained from Eqs. (2), (10) and 11) can be recast into a form that is more suitable for numerical calculations [23, 30, 31]:

$$
\begin{aligned}
A(\mathbf{k}, \omega)= & \sum_{n}\left[1+A_{n \mathbf{k}}^{\mathrm{S} 1}(\omega) *\right. \\
& \left.+\frac{1}{2} A_{n \mathbf{k}}^{\mathrm{S} 1}(\omega) * A_{n \mathbf{k}}^{\mathrm{S} 1}(\omega) *+\cdots\right] A_{n \mathbf{k}}^{\mathrm{QP}}(\omega) .
\end{aligned}
$$

Here we introduced the following quantities:

$$
\begin{aligned}
A^{\mathrm{QP}}(\omega) & =\frac{e^{\operatorname{Re} \Sigma^{\prime}(\varepsilon / \hbar)}}{\pi} \frac{|\operatorname{Im} \Sigma(\varepsilon / \hbar)|}{[\hbar \omega-\varepsilon-\operatorname{Re} \Sigma(\varepsilon / \hbar)]^{2}+[\operatorname{Im} \Sigma(\varepsilon / \hbar)]^{2}}, \\
A^{\mathrm{S} 1}(\omega) & =-\frac{1}{\pi} \frac{\operatorname{Im} \Sigma(\varepsilon / \hbar+\omega)-\operatorname{Im} \Sigma(\varepsilon / \hbar)-\hbar \omega \operatorname{Im} \Sigma^{\prime}(\varepsilon / \hbar)}{(\hbar \omega)^{2}}
\end{aligned}
$$

where we omitted the dependence on $n$ and $\mathbf{k}$, and the prime symbol denotes the first derivative. In the limit of a localized electron interacting with a plasmon bath, one may show that Eq. (12) reduces to the exact solution of the localized electron model given by Eq. (4). The application of this formalism to core and valence excitations of crystalline solids, on the other hand, involves several approximations such as neglecting recoil effects, that is the correlations between successive boson emission and reabsorption events. A detailed discussion of the range of validity of the cumulant expansion has been reported, for instance, in [21, 29, 32, 33.

As discussed by 34, the cumulant expansion has the advantage of introducing additional crossing and noncrossing Feynman diagrams that are neglected in the standard $G W$ and Migdal approximation for the selfenergy, and it results in an improved description of the electron-plasmon and electron-phonon interactions. The ab initio cumulant expansion approach is based on the evaluation of Eq. 12 employing either the $G W$ or Migdal self-energy. As discussed in Sec. VI, this formalisms also lends itself to describe the

The self-consistent solution of the Dyson's equation could in principle provide an alternative route to include additional diagrams beyond the $G W /$ Migdal approximation in the Green's function. However, while selfconsistent $G W$ has been shown to systematically improve the description of the quasiparticle energies of molecules and solids 35 37, the study of satellites has revealed that self-consistency leads to an unphysical renormalization of the satellite intensity which, ultimately, is expected to deteriorate the agreement with experiment 38]. Additional first-principles investigations would be needed to further explore this aspect.

\section{B. Plasmon satellites}

The concept of plasmons, collective fluctuations of the electron density, can be introduced based on a simple model of carrier dynamics for a homogeneous system (that is, a system characterized by a homogeneous electron density and a positively-charged ionic background) 
in which the quantum-mechanical character of the electrons is ignored. If an external perturbation as, for example, a homogeneous electric field is present, a displacement $\mathbf{x}$ of the electron density with respect to the positively charged ionic background is induced. The displaced electron density then generates an induced polarization $\mathbf{P}=-n e \mathbf{x}$, where $n$ is the average electron density and $e$ the electron charge, and an electric field $\mathbf{E}=-\mathbf{P} / \varepsilon_{0}$. Using Newton's law $m \ddot{\mathbf{x}}=-e \mathbf{E}$ with $m$ the electron mass, the classical equation of motion for the density displacement vector $\mathbf{x}$ may be rewritten as $\ddot{\mathbf{x}}+n e^{2} \mathbf{x} /\left(\varepsilon_{0} m\right)=0$. This model illustrates that the classical collective dynamics of electrons in solids can be approximately described by a harmonic oscillator with a characteristic frequency $\omega_{\mathrm{P}}=\sqrt{n e^{2} /\left(\varepsilon_{0} m\right)}$, the plasma frequency, which is independent of the perturbation and is determined exclusively by the intrinsic properties of the solid.

More generally, plasmons in solids may be excited at momenta $\mathbf{q}$ and frequencies $\omega_{\mathrm{P}}$ which correspond to vanishing real part of the macroscopic dielectric function $\epsilon_{\mathrm{M}}$ and sufficiently small imaginary part, that is:

$$
\epsilon_{\mathrm{M}}\left(\mathbf{q}, \omega_{\mathrm{P}}\right)=i \eta
$$

The macroscopic dielectric function $\epsilon_{\mathrm{M}}$ is related to the microscopic dielectric function $\epsilon$ via $\epsilon_{\mathrm{M}}^{-1}(\mathbf{q}, \omega)=$ $\left[\epsilon_{\mathbf{G}, \mathbf{G}^{\prime}}(\mathbf{q}, \omega)\right]_{\mathbf{G}=\mathbf{G}^{\prime}=0}^{-1}$, with $\mathbf{G}$ and $\mathbf{G}^{\prime}$ reciprocal lattice vectors and $\mathbf{q}$ in the first Brillouin zone. Whenever the condition expressed by Eq. 13. is satisfied, the system may support collective charge fluctuations even in the absence of an external driving field. In practice, the possibility of exciting plasmons is reflected by the emergence of sharp peaks in the loss function $L(\mathbf{q}, \omega)=$ $\operatorname{Im}\left[\epsilon_{\mathrm{M}}^{-1}(\mathbf{q}, \omega)\right]$ at the momenta and frequencies at which the macroscopic dielectric function $\epsilon_{\mathrm{M}}$ vanishes. The plasmon peaks in the loss function exhibit well defined energy-momentum dispersion relations. These structures are exemplified in Fig. 2 for the loss function of the homogeneous electron gas (HEG). If one neglects local-field effects, and thus assumes that the macroscopic and microscopic dielectric functions coincide, the plasmon energy is obtained by seeking the frequencies that satisfy the condition $v(\mathbf{q})^{-1}=\operatorname{Re} \chi_{0}(\mathbf{q}, \omega)$. For the HEG in the long-wavelength limit $(\mathbf{q} \rightarrow 0)$, this condition yields again the result $\omega_{\mathrm{P}}=\sqrt{e^{2} n /\left(\varepsilon_{0} m\right)}$.

The inspection of Eqs. (13) and (6) reveals that when the condition for the excitation of plasmons is satisfied, the screened Coulomb interaction $W_{\mathbf{G}, \mathbf{G}^{\prime}}(\mathbf{q}, \omega)=$ $v_{\mathbf{G}}(\mathbf{q})\left[\epsilon_{\mathbf{G}, \mathbf{G}^{\prime}}(\mathbf{q}, \omega)\right]^{-1}$ exhibits a pole at the plasmon energy. Correspondingly, one expects the $G W$ self-energy to encode information regarding electron-plasmon interaction.

To examine in more detail the inclusion of electronplasmon coupling effects in the $G W$ self-energy, we discuss below its connection with the electron-boson coupling model introduced in Sec. II. Using the condition given in Eq. (13) in combination with Eq. (6), the plasmonic contribution to the screened Coulomb interaction
$W$ can be disentangled from the other electronic contributions to the screening, such as electron-hole pairs. This idea, initially introduced for the homogeneous electron gas [1] and subsequently generalized to semiconductors 39, allows one to define a self-energy which stems exclusively from the coupling between electrons and plasmons. The resulting electron-plasmon self-energy can be recast into the following form [39]:

$$
\begin{aligned}
\Sigma_{n \mathbf{k}}^{\mathrm{eP}}= & \int \frac{d \mathbf{q}}{\Omega_{\mathrm{BZ}}} \sum_{m}\left|g_{m n}^{\mathrm{eP}}(\mathbf{k}, \mathbf{q})\right|^{2} \\
& \times\left[\frac{n_{\mathbf{q}}+f_{m \mathbf{k}+\mathbf{q}}}{\varepsilon_{n \mathbf{k}}-\varepsilon_{m \mathbf{k}+\mathbf{q}}+\hbar \omega_{\mathrm{P}}(\mathbf{q})+i \eta}\right. \\
& \left.+\frac{n_{\mathbf{q}}+1-f_{m \mathbf{k}+\mathbf{q}}}{\varepsilon_{n \mathbf{k}}-\varepsilon_{m \mathbf{k}+\mathbf{q}}-\hbar \omega_{\mathrm{P}}(\mathbf{q})+i \eta}\right]
\end{aligned}
$$

where the coefficients $g_{m n}^{\mathrm{eP}}(\mathbf{k}, \mathbf{q})$ are the electron-plasmon scattering matrix elements between the initial state $\psi_{n \mathbf{k}}$ and the final state $\psi_{m \mathbf{k}+\mathbf{q}}$ and are given by:

$g_{m n}^{\mathrm{eP}}(\mathbf{k}, \mathbf{q})=\left[\frac{\varepsilon_{0} \Omega}{e^{2} \hbar} \frac{\partial \epsilon(\mathbf{q}, \omega)}{\partial \omega}\right]_{\omega_{\mathrm{P}}(\mathbf{q})}^{-\frac{1}{2}} \frac{1}{|\mathbf{q}|}\left\langle\psi_{m \mathbf{k}+\mathbf{q}}\left|e^{i \mathbf{q} \cdot \mathbf{r}}\right| \psi_{n \mathbf{k}}\right\rangle$,

with $\Omega$ being the volume of the unit cell. Equation 14 has the form of an electron-boson coupling self-energy in the Migdal approximation [see Eq. (7)], which may alternatively be derived from an electron-boson coupling Hamiltonian of the form:

$$
\hat{H}^{\mathrm{eP}}=\sum_{n m} \sum_{\mathbf{k}, \mathbf{q}} g_{n m}^{\mathrm{eP}}(\mathbf{k}, \mathbf{q}) \hat{c}_{m \mathbf{k}+\mathbf{q}}^{\dagger} \hat{c}_{n \mathbf{k}}\left(\hat{b}_{\mathbf{q}}+\hat{b}_{-\mathbf{q}}^{\dagger}\right) .
$$

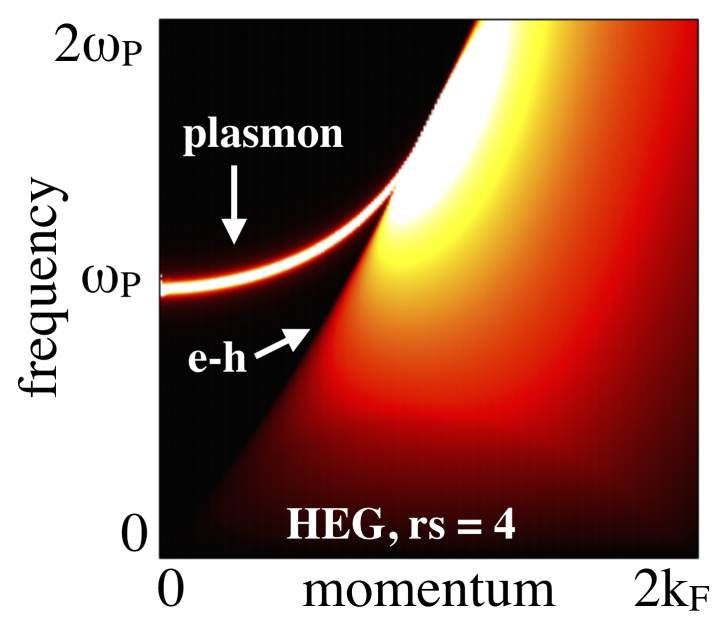

FIG. 2. The loss function of the HEG for a Wigner-Seitz radius $r_{s}=4$. The plasmonic structures in the loss function follow a characteristic parabolic dispersion which, for momenta smaller than a critical momentum $q_{\mathrm{c}}$, is well separated from the continuum of electron-hole excitations (e-h). For $q>q_{\mathrm{c}}$, the plasmons are damped by the interaction with electron-hole pairs (Landau damping). 
Here $\hat{b}_{-\mathbf{q}}^{\dagger}\left(\hat{b}_{\mathbf{q}}\right)$ and $\hat{c}_{m \mathbf{k}+\mathbf{q}}^{\dagger}\left(\hat{c}_{n \mathbf{k}}\right)$ are the boson and fermion creation (destruction) operators, respectively. The localized electron model of Eq. (1) is recovered from Eq. (16) by (i) replacing the Bloch energies $\varepsilon_{n \mathbf{k}}$ with a single nondispersive energy and (ii) neglecting the k-dependence of the electron-boson coupling matrix elements. This result indicates that the $G W$ self-energy accounts for the coupling between electrons and plasmons. However, at variance with the localized electron model which could be solved exactly, here the electron-plasmon interaction is treated only at first-order in the interaction strength, which corresponds to the Migdal approximation in the ordinary electron-boson coupling theory.

The inclusion of electron-plasmon coupling in the $G W$ theory is reflected by the emergence of plasmon satellites in the spectral function, which are analogous to the satellite features discussed in Sec. II. In fact, in the presence of plasmons, the frequency dependence of the self-energy typically presents a pole, which may produce additional satellite structures in the spectral function signaling the coupling to plasmons.

Two clear shortcomings emerge when evaluating the spectral function within the $G W$ approximation, and limit its predictive power for the description of satellites in PES: (i) the energy difference between the satellite and the quasiparticle peak is typically overestimated by a factor of 1.5 with respect to photoemission experiments, and (ii) the $G W$ approximation may erroneously predict the formation of spurious plasmaron peaks, which stem from additional solutions of the quasiparticle equation and often result in an overestimation of satellite intensities. The concept of plasmaron was initially introduced by [1] as a new quasiparticle state emerging from the strong coupling between electrons and plasmons. Later studies, however, revealed that plasmaron peaks are an artifact of the $G W$ approximation and, in fact, they disappear when one resorts to a more accurate level of theory [3]. These issues can be illustrated by using a simplified model for the $G W$ self-energy: $\Sigma(\omega)=\alpha\left(\hbar \omega-\varepsilon+\omega_{P}+i \eta\right)^{-1}$. This expression is derived from Eq. (6) by (i) assuming non-dispersive electron energies, (ii) replacing the oscillator strengths by $\delta$ functions, (iii) using a plasmon-pole model for the dielectric function in the form $\epsilon^{-1}(\omega)=1+\tilde{\omega} /\left(\omega^{2}-\omega_{\mathrm{P}}^{2}+i \eta\right)$, and (iv) carrying out the frequency integration analytically.

As shown in Fig. 3, the self-energy exhibits a pole at frequencies around $\omega=\hbar^{-1}\left(\varepsilon-\hbar \omega_{\mathrm{P}}\right)$, which may lead to additional unphysical solutions of the quasiparticle equation when $\hbar \omega-\epsilon=\operatorname{Re} \Sigma(\omega)$ as shown in panel (a), or to a minimum in $\hbar \omega-\epsilon-\operatorname{Re} \Sigma(\omega)$ resulting in a weak satellite, as shown in panel (b). In both cases the spectral function is characterized by the emergence of satellites, however their binding energy is blue-shifted with respect to the energy $\epsilon-\hbar \omega_{\mathrm{P}}$ at which satellites are typically observed in PES experiment. As we will discuss in Sec. IV] the combination of the $G W$ approximation with the cumulant expansion approach $(G W+\mathrm{C})$ allows to successfully address these issues and recover an energy separation be-

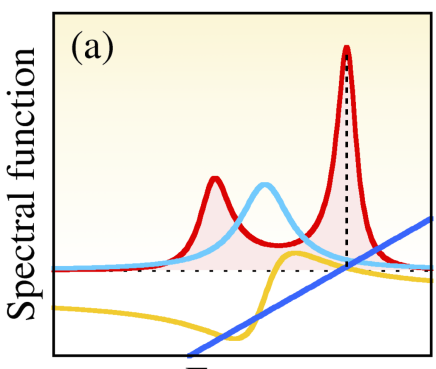

Energy

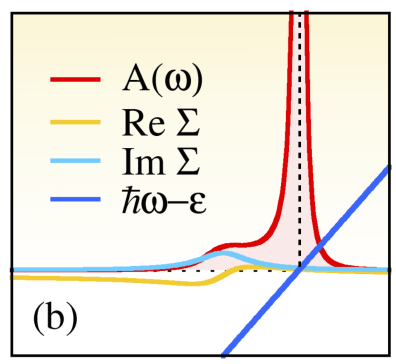

Energy
FIG. 3. Spectral function for a model self-energy in the strong (a) and weak (b) coupling regime. Quasiparticle peaks are marked by vertical dashed lines and correspond to the intersection between $\operatorname{Re} \Sigma(\omega)$ and $\hbar \omega-\varepsilon$.

tween satellite and quasiparticle peaks that agrees well with PES measurements for a broad class of materials.

\section{Polaron satellites}

Similarly to the case of the interaction with plasmons, the coupling between electrons and phonons may give rise to satellite structures in the spectral function of semiconductors and insulators, which are the signature of the dressing of the electronic quasiparticles as polarons. The formation of polarons is typically linked to the polarization of the lattice induced by longitudinal optical (LO) phonons. In other words, in polar semiconductors and insulators the fluctuations of the ionic positions corresponding to LO phonons at long wavelength generate macroscopic electric fields which can couple strongly to electrons and holes. This long-range interaction is known as Fröhlich coupling. The Fröhlich model strictly describes the interaction of a conduction electron in a parabolic band with LO phonons of constant energy $\hbar \omega_{\mathrm{LO}}$, in an isotropic and uniform medium [40. Under these assumptions, the electron-phonon matrix element does not depend on the band index and electron momentum, and it takes the form:

$$
g_{\mathrm{F}}(\mathbf{q})=\frac{i}{|\mathbf{q}|}\left[\frac{e^{2}}{4 \pi \varepsilon_{0}} \frac{4 \pi}{\Omega} \frac{\hbar \omega_{\mathrm{LO}}}{2}\left(\frac{1}{\epsilon_{\infty}}-\frac{1}{\epsilon_{\mathrm{s}}}\right)\right]^{1 / 2}
$$

where $\epsilon_{\mathrm{S}}$ is the total static permittivity (or dielectric constant) and $\epsilon_{\infty}$ is the optical dielectric constant, that is $\epsilon_{M}(\mathbf{q}=0, \omega=0)$. The matrix element in Eq. (17) is often expressed in terms of a dimensionless parameter $\alpha$ which is referred to as the Fröhlich coupling constant:

$$
\alpha=\frac{e^{2}}{\hbar}\left(\frac{m_{\mathrm{b}}}{2 \hbar \omega_{\mathrm{LO}}}\right)^{1 / 2}\left(\frac{1}{\epsilon_{\infty}}-\frac{1}{\epsilon_{\mathrm{s}}}\right),
$$

with $m_{\mathrm{b}}$ the band effective mass of the conduction electron. The Fröhlich Hamiltonian has the form in Eq. (16) after substituting $g_{m n}^{\mathrm{eP}}$ with $g_{\mathrm{F}} \delta_{m n}$, and it is thus historically representative of the general problem of a fermionic 
particle interacting with a boson field. Depending on the value of $\alpha$, i.e. on the strength of the coupling, the Fröhlich self-energy produces a spectral function that usually exhibits satellite replica of the main quasiparticle band.

First-principles calculations of electron-phonon selfenergies and ARPES spectra to capture polaronic effects are limited by the almost prohibitive computational cost of sampling the singular behavior of the matrix elements for small phonon wavevectors. A procedure that enables accurate calculations of the electron-phonon coupling in the presence of Fröhlich interaction at a reduced computational cost has been reported by [41, 42]. This is achieved via the separation of the long-range, singular part of the electron-phonon matrix element and of the short-range part. The long-range part $g^{\mathcal{L}}$ constitutes the generalization of the Fröhlich matrix element to multiple, anisotropic electronic bands and phonon modes, and reads:

$$
\begin{aligned}
g_{m n \nu}^{\mathcal{L}}(\mathbf{k}, \mathbf{q})= & i \frac{4 \pi e^{2}}{\Omega} \sum_{\kappa}\left(\frac{\hbar}{2 M_{\kappa} \omega_{\mathbf{q} \nu}}\right)^{1 / 2} \\
& \times \sum_{\mathbf{G}, \mathbf{q}+\mathbf{G} \neq 0} \frac{(\mathbf{q}+\mathbf{G}) \cdot \mathbf{Z}_{\kappa}^{*} \cdot \mathbf{e}_{\kappa \nu}(\mathbf{q})}{(\mathbf{q}+\mathbf{G}) \cdot \boldsymbol{\epsilon}_{\infty} \cdot(\mathbf{q}+\mathbf{G})} \\
& \times\left\langle\psi_{m \mathbf{k}+\mathbf{q}}\left|e^{i(\mathbf{q}+\mathbf{G}) \cdot\left(\mathbf{r}-\boldsymbol{\tau}_{\kappa}^{0}\right)}\right| \psi_{n \mathbf{k}}\right\rangle,
\end{aligned}
$$

where $\mathbf{Z}_{\kappa}^{*}$ is the Born effective charge tensor of atom $\kappa$ in the unit cell, $M_{\kappa}$ the atomic mass and $\mathbf{e}_{\kappa \nu}(\mathbf{q})$ a phonon eigenvector. If combined with the Wannier-Fourier interpolation technique of [43, Eq. (19) enables accurate calculations of polaron satellites.

As in the case of the $G W$ method, the calculation of the spectral function including electron-phonon coupling in the Migdal approximation suffers from two main shortcomings. First, it produces only a single polaronic satellite rather than a Lang-Firsov series as shown by the model of Eqs. (1) and (4), and as measured in experiments. Second, its energy separation from the main quasiparticle peak is larger than the characteristic LO phonon energy. As we will illustrate in Sec. IV] and $\mathrm{V}$. the cumulant expansion method can successfully be employed to improve the description of satellites. While this method has been mostly used in combination with the $G W$ approximation to study plasmon satellites, it can also naturally be applied in the context of polaronic systems, since the theory stems from the exact solution of an electron-boson coupling Hamiltonian of the Fröhlich type [3, 44. The formalism corresponds to the one presented in Sec. III A, with the Migdal electron-phonon self-energy used as a seed.

\section{PLASMON SATELliteS IN METALS AND SEMICONDUCTORS}

First-principles calculations of plasmon satellites based on the $G W+\mathrm{C}$ approach have first been performed by 30 . for metallic sodium and aluminum. The integrated photoemission spectroscopy experiment on sodium by [45] revealed, besides a quasiparticle peak centered at a binding energy of $1 \mathrm{eV}$ which corresponds to the excitation of photo-holes in the valence band, two broader and less intense satellite peaks blue-shifted with respect to the quasiparticle peak by 6 and $12 \mathrm{eV}$ respectively. These energies are compatible with multiples of the plasma energy of sodium $\hbar \omega_{\mathrm{P}} \simeq 5.9 \mathrm{eV}$, suggesting that the satellites arise from the excitation of one and two plasmons. At variance with the $G W$ results, which overestimate the energy and intensity of the satellites, the spectral function of Na obtained from the $G W+\mathrm{C}$ approach and shown in Fig. 4(a) improves significantly the agreement with the experiment. Additionally, the $G W+\mathrm{C}$ approach lends itself to describe also processes in which more than one plasmon are excited, and captures the emergence of a series of satellite peaks spaced by the plasmon energy. On the other hand, only one satellite is obtained within the $G W$ approximation, reflecting the fact that multiplasmon processes are neglected.

Subsequently, photoemission satellites have been measured in the photoemission spectra of graphene and the identification of these features has been supported by theoretical calculations of the self-energy and spectral function for linearly-dispersive bands [5]. Satellites in semiconductors have first been investigated from first principles by [46] for the case of silicon. In analogy with metals, also photoemission measurements of semiconductors may exhibit a series of satellites (Fig. 4(b)) with an energy separation that is compatible with the plasma energy. In this case, however, plasmons are generally characterized by a smaller oscillator strength, which is reflected by the lower intensity of the satellite peaks.

For silicon, the $G W$ approximation yields a single satellite blue-shifted by $\sim 22 \mathrm{eV}$ with respect to the quasiparticle peak, which is incompatible with the plasma energy $\hbar \omega_{\mathrm{P}}=16.6 \mathrm{eV}$ and with the experimental observations. On the other hand, when vertex corrections are included via the $G W+\mathrm{C}$ approach the energy of the plasmon satellite peak is in good agreement with experiment. Some discrepancies between theory and experiment still remain, namely: (i) the intensity of the satellite peak is underestimated; (ii) the relative intensity between the different substructures of the quasiparticle peak differs from the experimental result; (iii) experiments present a featureless background signal that increases with the electron binding energy and that is not captured by theory.

The points (i)-(iii) are directly related to the interpretation of the spectral function as a photoelectron current, an approximation that is typically referred to as sudden approximation [9, 47. In practice, the sudden approximation assumes that all electrons are equally likely to interact with an incoming photon, and that after photoexcitation the electrons do not interact further with the sample. These assumptions neglect the scattering crosssection effects due to the different orbital symmetries, 

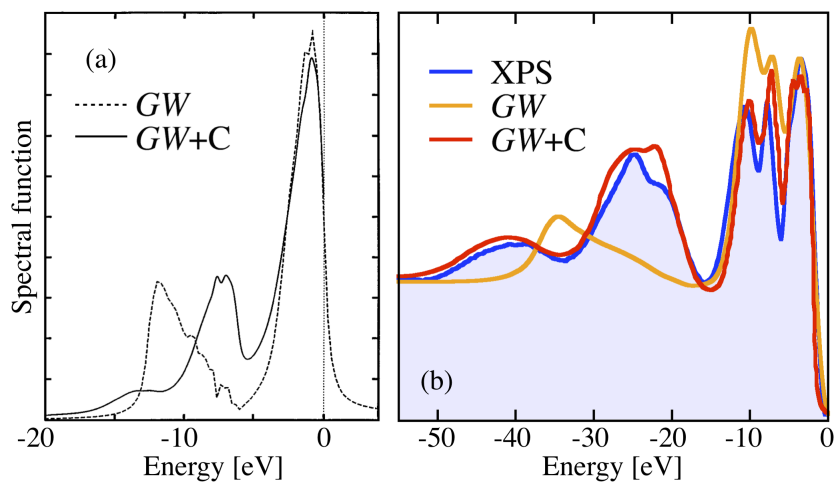

FIG. 4. (a) Cumulant expansion for Na. (b) Cumulant expansion for silicon. Reproduced with permission from 30 , 46.

and the additional energy losses that photo-electrons may undergo after emission from the initial state. The issues mentioned in (i)-(iii) could be improved by adopting a picture of the photoemission process that goes beyond the sudden approximation, e.g. by explicitly accounting for extrinsic losses, background signal, and cross-section effects. In this way, a quantitatively accurate description of satellites in semiconductors may be achieved [48.

Inspection of the first satellite peak in Fig. 4(b) indicates that the plasmon-induced spectral features of silicon are characterized by a substructure - in this case a central peak and two shoulders observed in both theory and experiments - that resembles the density of states (DOS) of the ordinary quasiparticle bands. To understand the origin of these features it is convenient to recall the concept of Van Hove singularities from the quantum theory of solids. The density of states $J$ for a set of Bloch electrons can be expressed as:

$$
J(\omega)=\frac{1}{4 \pi^{3}} \sum_{n} \int_{S(\hbar \omega)} d S_{\mathbf{k}} \frac{1}{\left|\nabla_{\mathbf{k}} \varepsilon_{n \mathbf{k}}\right|}
$$

where the integral is performed over the isosurfaces in k-space with energy $\hbar \omega$, denoted by $S(\hbar \omega)$. If for a given energy $\hbar \omega$, the isosurface $S(\hbar \omega)$ contains a crystal momentum for which the electron velocity vanishes $\left(v_{n \mathbf{k}}=\nabla_{\mathbf{k}} \epsilon_{n \mathbf{k}} / \hbar=0\right)$, the divergence of the integrand in Eq. 20 leads to a sharp structure in $J(\omega)$, referred to as a Van Hove singularity. Peaks in the DOS may thus be attributed to regions of the Brillouin zone in which electronic bands are flat $\left(\nabla_{\mathbf{k}} \varepsilon_{n \mathbf{k}} \simeq 0\right)$. These structures are clearly visible in PES experiments of silicon for binding energies between 0 and $-15 \mathrm{eV}$ [Fig. 5(b)] and in the DOS obtained from DFT calculations in the local density approximation [Fig. 5(c)]. The structure of plasmon satellites measured in PES also exhibits a substructure of peaks and shoulders that resembles the Van Hove singularities, as it can be noted when comparing it with the DOS of the ordinary quasiparticle bands red-shifted by the plasmon energy [Fig. [5(b)-(c)]. This suggests that the plasmon satellites observed in integrated PES also stem from the average over the Brillouin zone of spectral features that are characterized by well-defined energymomentum dispersion relations.

This hypothesis has been been verified by firstprinciples calculations of the angle-resolved spectral function of silicon in the $G W+\mathrm{C}$ approach [18, which revealed that electron-plasmon interaction leads to the emergence of plasmonic polaron bands, that is, plasmoninduced replica of the valence band structure of semiconductors red-shifted by the plasmon energies. These features are illustrated for silicon in Fig. 5(a). As compared to the quasiparticle bands, plasmonic polaron bands are less intense due to the small oscillator strength of plasmon in semiconductors, and broadened out by lifetime effects. The existence of plasmonic polaron bands has been corroborated by further theoretical and experimental investigations of the ARPES spectrum of silicon for binding energies up to $40 \mathrm{eV}$ by [20]. Overall, the dispersive character of plasmon-induced features in ARPES indicates that plasmon satellites in integrated PES may be interpreted as Van Hove singularities which arise from the flattening of the plasmonic polaron bands at specific regions in the Brillouin zone.

\section{POLARON SATELLITES IN DOPED SEMICONDUCTORS}

Low-energy satellites have recently been observed by ARPES experiments in doped oxides. These systems constitute an ideal playground for the study of polaron physics. In particular, satellite replicas were measured for $n$-doped $\mathrm{TiO}_{2}$ [6, $\mathrm{SrTiO}_{3}$ [4] and monolayer FeSe on $\mathrm{SrTiO}_{3}$ [50]. Evidence of Fröhlich polarons was found also from the investigation of two-dimensional (2D) electronic states at the surfaces or interfaces of oxides, with the most studied case being the 2D electron gas (2DEG) formed at the surface of $\mathrm{SrTiO}_{3}$ [7, 51, 52. Other notable examples are the $2 \mathrm{DEG}$ at the interface between $\mathrm{SrTiO}_{3}$ and $\mathrm{LaAlO}_{3}$ [53] and on the surface of $\mathrm{ZnO}$ [54]. The experiments also show a remarkable evolution of the carriers with doping concentration, from polarons to a Fermi liquid weakly coupled to phonons [6, 7].

Calculations of the spectral function using model selfenergies or the localized electron model of Eq. (4) have been performed for some of these systems, for example in [6, 50, 51, 55]. Fully $a b$ initio calculations showing satellite band replica were first reported for the insulating compounds $\mathrm{MgO}$ and LiF by [56], subsequently exploring also the effect of the cumulant expansion method [57. First-principles calculations of ARPES spectra in doped materials including polaronic effects were carried out by [23. for the prototypical case of anatase $\mathrm{TiO}_{2}$ by using the methods presented in Sec. III. Given that the crystals are doped, an important element that needs to be taken into account when performing $a b$ initio calculations is the presence of additional charges in the con- 


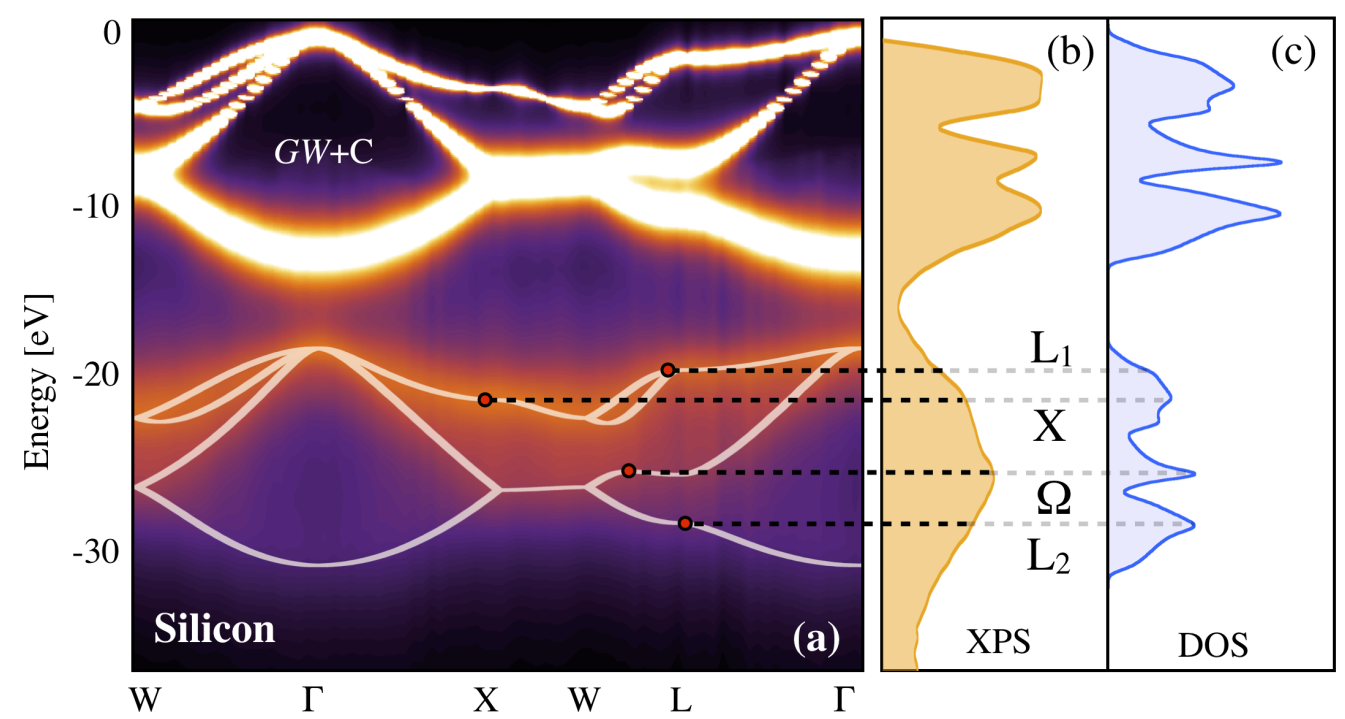

FIG. 5. (a) Theoretical calculations of the plasmonic polaron band of silicon, based on the $G W$ plus cumulant expansion approach, adapted from 21. (b) Integrated X-ray photoemission spectrum (XPS) of silicon from [46]. (c) Density of states of silicon from a density functional theory calculation alongside with a replica of the full DOS red-shifted by the plasmon energy $\hbar \omega_{\mathrm{P}}=16.6 \mathrm{eV}$.

duction band. Since the systems of interest are degenerate and present well-defined Fermi surfaces, doping can be treated in the rigid-band approximation, that is by placing the Fermi level inside the conduction or valence band of the pristine system. Moreover, the added carriers provide an additional source of screening of the electronphonon interactions. This effect is critical especially in the case of polar coupling, where the screening of the macroscopic electric field created by the LO phonons can change dramatically the strength of the Fröhlich interaction [15. This aspect can be understood by considering the simple Thomas-Fermi screening model, which describes the static response of a homogeneous electron gas at small wavevectors: $\epsilon_{\mathrm{TF}}(\mathbf{q})=1+q_{\mathrm{TF}}^{2} /|\mathbf{q}|^{2}$, with $q_{\mathrm{TF}}=\sqrt{2 e^{2} n /\left(\varepsilon_{0} \epsilon_{\infty} E_{\mathrm{F}}\right)}\left(E_{\mathrm{F}}\right.$ is the Fermi energy). From the wavevector dependence of $\epsilon_{\mathrm{TF}}(\mathbf{q})$ it follows immediately that the screened matrix element, $g_{\mathrm{F}}(\mathbf{q}) / \epsilon_{\mathrm{TF}}(\mathbf{q})$, no longer exhibits a singularity at long wavelength. This model is valid in the adiabatic limit where the doped carriers instantaneously follow the atomic motion. In a more accurate description, the timescale of the electronic response is dictated by the plasma frequency of the doped carriers, $\omega_{\mathrm{P}}=\sqrt{n e^{2} /\left(\varepsilon_{0} \epsilon_{\infty} m_{\mathrm{b}}\right)}$ in the HEG model 58.

To capture the evolution of the electron-phonon coupling and of the polaronic features with doping, the electron-phonon matrix element needs to be screened by the dynamical dielectric function evaluated at the phonon energies, that is $g_{m n \nu}^{\mathrm{NA}}(\mathbf{k}, \mathbf{q})=g_{m n \nu}(\mathbf{k}, \mathbf{q}) / \epsilon_{\mathrm{RPA}}\left(\mathbf{q}, \omega_{\mathbf{q} \nu}+\right.$ $\left.i / \tau_{n \mathbf{k}}\right)$ [15, 23]. The superscript NA indicates that retardation effects are taken into account by using this nonadiabatic matrix element, and $\hbar / \tau_{n \mathbf{k}}$ is the electron lifetime near the band edge, which can approximately be taken to be constant. In practical calculations the dynamical screening arising from the doped carriers can be computed analytically using the RPA dielectric function for a homogeneous electron gas with the same density $n$, which is known as the Lindhard function [59].

In Fig. 6 we show the ARPES spectra acquired for $n$ doped anatase $\mathrm{TiO}_{2}$ by [6], and we compare them with the first-principles calculations performed by [23]. The spectra for the first two doping levels exhibit a satellite about $0.1 \mathrm{eV}$ below the main parabolic band, and a second very dim satellite at another $0.1 \mathrm{eV}$ higher binding energy. Since the energy separation of the band replica is compatible with the high-energy $E_{u}$ LO phonon of anatase $\mathrm{TiO}_{2}$, these satellites were attributed to polaronic effects. At the highest doping, on the other hand, the satellites disappear and are replaced by band structure kinks near a binding energy of $0.1 \mathrm{eV}$. All the spectral features and their evolution with doping are reproduced by the calculations, thus confirming the transition from a polaronic to a Fermi liquid picture, and demonstrating the success of the first-principles methods used to investigate quasiparticle spectra. From the calculated ARPES spectra the electron-phonon coupling strength $\lambda$ was extracted, by using the ratio between the Fermi velocities of the bare band and of the dressed band [15. The results are reported in Fig. 7, together with an analysis of the energy scales at play. The study showed that the crossover from polarons to a weakly-coupled Fermi liquid and, correspondingly, from satellite replica to band structure kinks, occurs when the plasma frequency of the carriers becomes of the order of the LO phonon frequency. In fact, in the polaronic regime, corresponding to $\omega_{\mathrm{P}}<\omega_{\mathrm{LO}}$, the carriers are too slow to screen the longrange electric field generated by the $E_{u}$ phonon vibrations. In this case satellites appear in the spectra, and the electron-phonon coupling strength is approximately inde- 
(a)
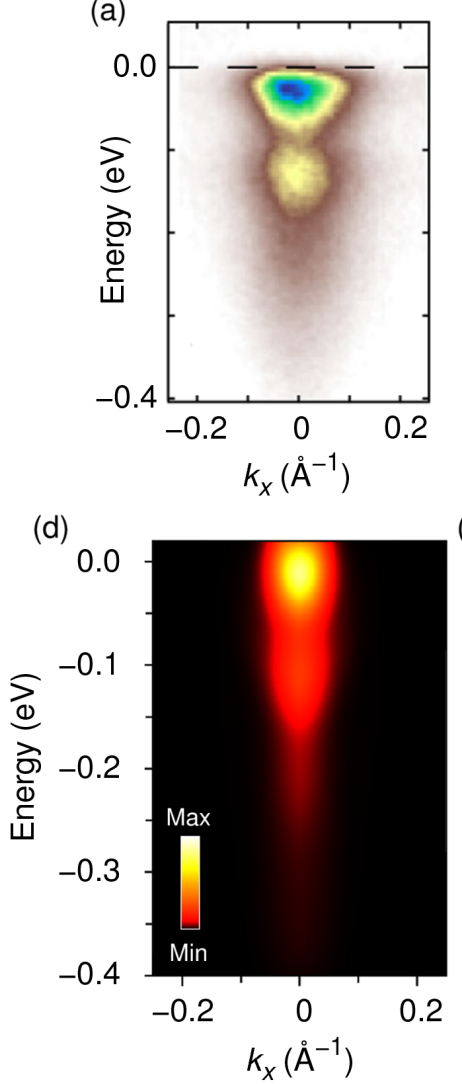

(b)

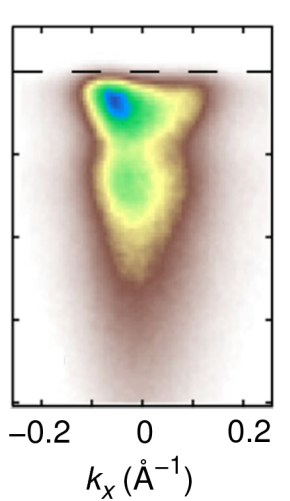

(e)

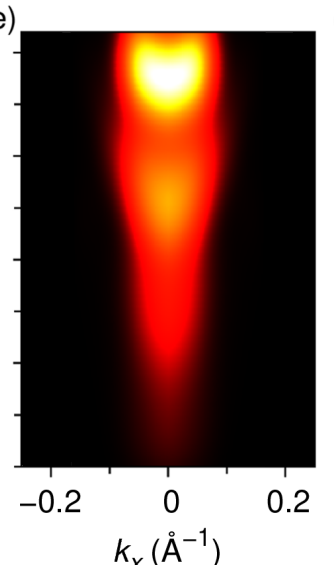

(c)

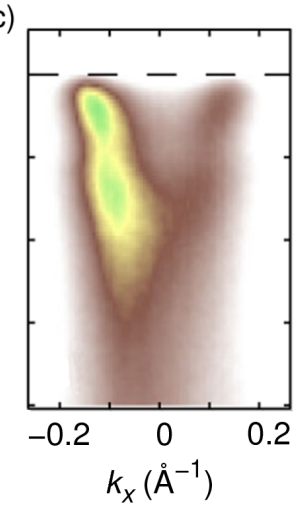

(f)

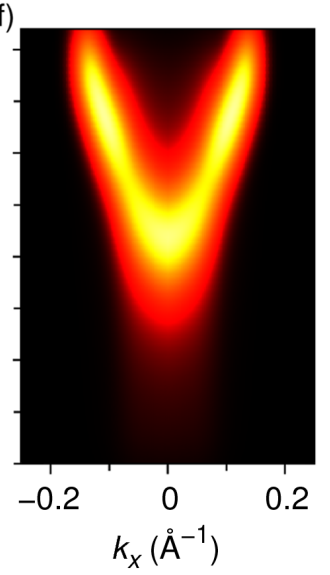

FIG. 6. ARPES spectra of $n$-doped anatase $\mathrm{TiO}_{2}$ on samples with doping concentrations $5 \times 10^{18} \mathrm{~cm}^{-3}$ (a), $3 \times 10^{19} \mathrm{~cm}^{-3}$ (b) and $3.5 \times 10^{20} \mathrm{~cm}^{-3}$, taken from [6]. The corresponding first-principles spectra from 23 are shown in panels (d)-(f). The calculated spectral functions were multiplied by the Fermi-Dirac distribution at the experimental temperature $(T=20 \mathrm{~K})$ and were convoluted with Gaussian masks of widths $25 \mathrm{meV}$ and $0.015 \AA^{-1}$ in order to account for the experimental resolution in energy and momentum, respectively.

pendent of doping. When $\omega_{\mathrm{P}}>\omega_{\mathrm{LO}}$, in the Fermi liquid regime, the Fröhlich coupling is strongly suppressed, with the polaron satellites gradually replaced by kinks. Correspondingly, the coupling strength decreases. This first-principles analysis indicated that the interplay between lattice vibrations and plasma oscillations can have a strong impact on the polaronic properties of charge carriers in doped oxides.

\section{HYBRID PLASMON-PHONON SATELLITES}

Interestingly, the effects of electron-phonon and electron-plasmon interactions can be readily combined within first-principles calculations if the sum of the relative self-energies [Eqs. (7) and (14]] is included in the calculation of the spectral function, which can thus contain both plasmon and polaron satellite features. This concurrence of plasmon and polaron satellites has been observed experimentally in the case of the ferromagnetic semiconductor $\mathrm{EuO}$, and confirmed by first principles calcula- tions 60]. Experimental ARPES spectra for Gd-doped $\mathrm{EuO}$ are reported in Fig. 8(a)-(c) for three different doping concentrations, showing the bottom of the conduction band centered at the $\mathrm{X}$ point of the Brillouin zone. The energy distribution curves (EDCs) at the conduction band minimum for several dopings are reproduced in Fig. $8(\mathrm{~g})$, and they clearly show a shoulder peak whose energy separation with respect to the main quasiparticle band increases with carrier concentration.

Such a satellite peak is not resolved above a carrier density $n \approx 10^{20} \mathrm{~cm}^{-3}$, whereas at low carrier concentration $\left(n \approx 10^{18} \mathrm{~cm}^{-3}\right)$ two additional satellites can be distinguished. The spectra calculated with the cumulant expansion method including electron-phonon and electron-plasmon coupling on the same footing are presented in Fig. 8(d)-(f), and they reproduce the features seen in the experiment. In particular, the calculations confirmed that for the lowest doping concentration the series of satellites is mainly due to phonon excitations, with the LO phonon energy of $\mathrm{EuO}$ being compatible with the peak separation energy of about $56 \mathrm{meV}$. Moving to higher dopings, the polar electron-phonon cou- 


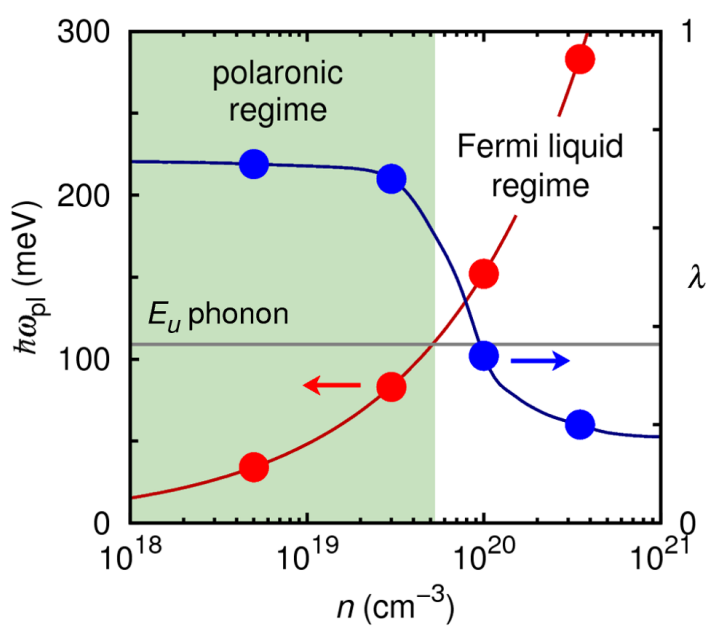

FIG. 7. Polaronic and Fermi liquid regimes in $n$-doped anatase $\mathrm{TiO}_{2}$, from 23]: the red disks indicate the plasma energy at each doping level, the blue disks indicate the electronphonon coupling strength $\lambda$. The blue line is a guide to the eye, while the red line represents the relation between the plasma energy and the doping density in the homogeneous electron gas. The horizontal line is the energy of the LO $E_{u}$ phonon of anatase $\mathrm{TiO}_{2}, 109 \mathrm{meV}$.

pling is gradually suppressed by the free-carrier screening, and the satellite peak shifts to higher binding energies. Since the plasma energy increases as the square-root of the carrier density, this finding constitutes a fingerprint of the coupling of electrons to plasmonic excitations of the conduction electrons. The first-principles calculations confirmed that the renormalization of the spectral properties at higher dopings is due to the interplay between electron-phonon and electron-plasmon coupling, and that in particular the low-energy broad satellite seen in Fig. 8(c),(f) is due to plasmonic excitations [60].

We remark that the calculations and methods presented so far neglect the effects of mutual renormalization between plasmon and phonon modes, which can arise when the frequency of plasmon and phonon oscillations are of the same order [61, 62]. The inclusion of these effects entirely from first principles represents one of the challenges still open in the investigation of the spectral properties of doped systems.

\section{CONCLUSIONS}

The emergence of satellites in photoemission spectroscopy is a universal manifestation of electron-boson interactions in solids. The origin of satellites can be ascribed to the excitation of different types of bosonic modes such as valence plasmons, extrinsic plasmons, or polar phonons. These spectral features have thus far been observed in metals, semiconductors, and highlydoped oxides. Despite the diversity of the physical processes that underpin the satellite formation, and the broad energy scales (from 50-100 meV up to $15-20 \mathrm{eV}$ ), many-body perturbation theory provides a unified framework for their description. In combination with standard approximations for the electron-electron and electronphonon self-energies, the cumulant expansion approach is a powerful tool for investigating the emergence of spectral fingerprint of electron-boson coupling in solids.

The study of satellites in solids has thus far provided valuable insight into the many-body interactions between electrons, plasmons, and phonons. Recent work in this area has demonstrated that first-principles techniques have reached an accuracy sufficient to even precede experiments in discovering new hallmarks of the coupling between electrons and bosons. The emergence of satellites in photoemission spectroscopy is just one facet of the many effects that electron-boson interaction may induce. The recent findings discussed in this chapter call for a systematic investigation of the influence of low-energy plasmons on the formation of photoemission kinks, waterfall effects, as well as novel mechanisms of superconductive pairing. Furthermore, other spectroscopic techniques, such as absorption, electron energy loss, or timeresolved spectroscopies, provide less explored tools for investigating the coupling between electrons, plasmons, and phonons. Highly-doped oxides constitute a particularly exciting playground for exploring the influence of these phenomena on the opto-electronic properties and possible opportunities for exploiting these new emergent properties. In these compounds, the interplay of carriers, extrinsic plasmons, and polar phonons, induces complex spectral features that reflect the simultaneous excitation of plasmon and phonon modes and that are highly tunable via the carrier concentration.

In conclusion, the last few years have witnessed a remarkable increase in the accuracy of theoretical techniques for the description of the excited-state phenomena from first principles. These advances, alongside with a relentless increase in experimental resolution, are contributing to strengthen the synergy between theoretical and experimental research, providing numerous opportunities to unveil and understand unexplored forms of fermion-boson coupling in quantum matter.

\section{ACKNOWLEDGMENTS}

FC thanks Matteo Guzzo for sharing the data illustrated in Fig. 4. The authors gratefully acknowledge support from the Leverhulme Trust (Grants PLP-2015-144 and RL-2012-001), the Graphene Flagship (Horizon 2020 Grant No. 785219 - GrapheneCore2), and the EPSRC (Grant No. EP/M020517/1). 

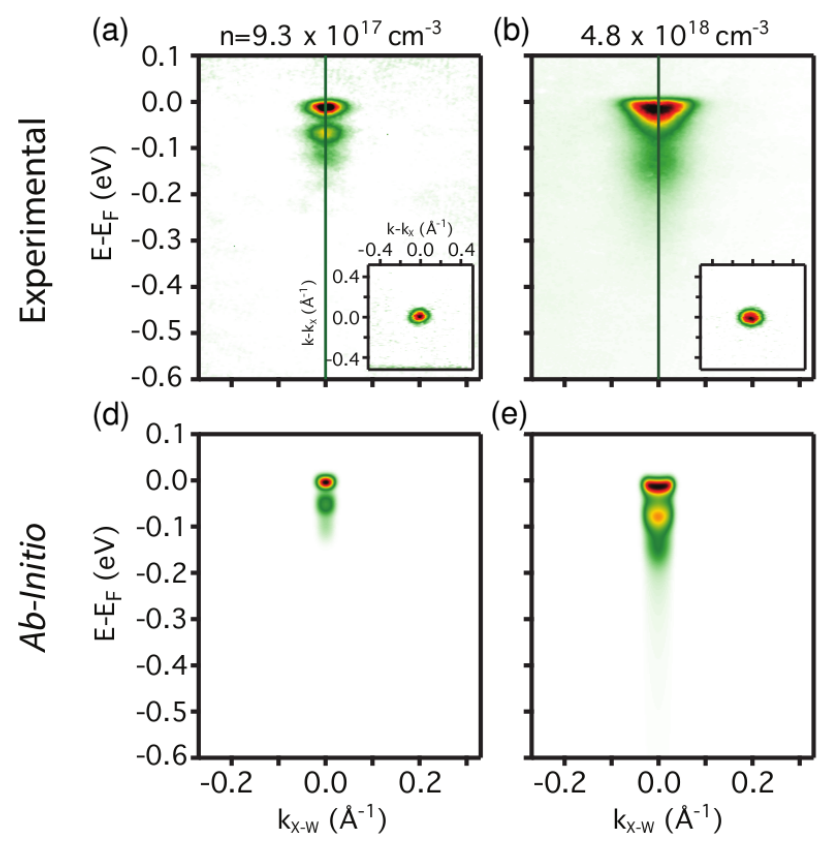

(e)

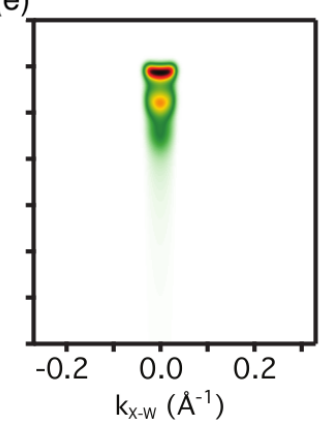

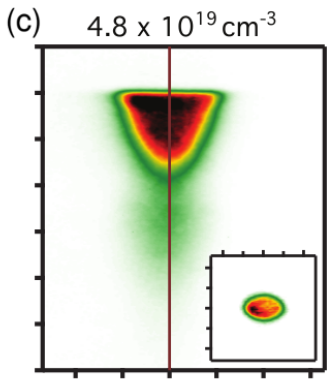

(f)

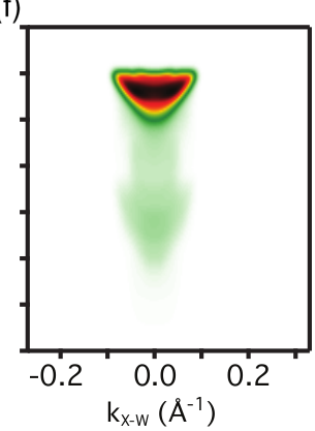

(g)

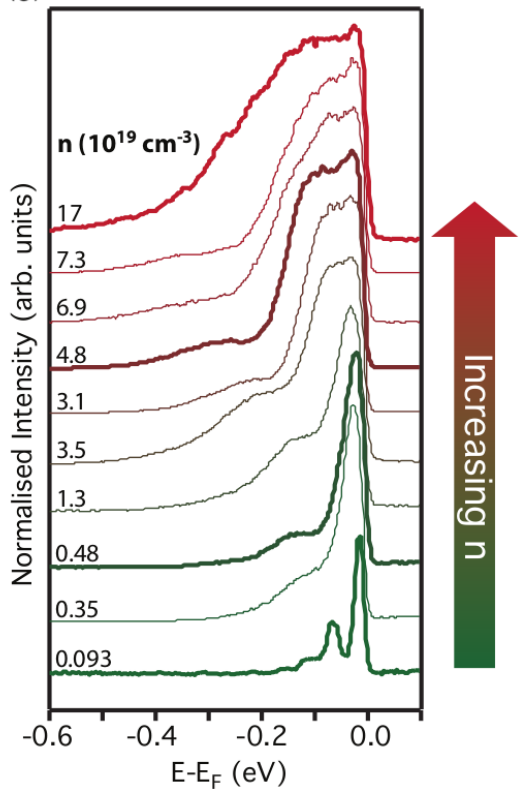

FIG. 8. (a)-(c) Measured ARPES spectra of EuO samples with increasing carrier concentrations as indicated on top of each panel, with the Fermi surface contours shown in the insets. The corresponding first-principles data are shown in (d)-(f). To directly compare with the experiments, the calculated spectral functions were convoluted with two Gaussian masks of widths $20 \mathrm{meV}$ and $0.015 \AA^{-1}$, and integrated along the out-of-plane direction $k_{z}$. (g) Measured energy distribution curves taken at $k=k_{\mathrm{X}}$ (conduction band minimum) for different doping levels. Figure adapted from 60.

[1] B. I. Lundqvist, "Single-particle spectrum of the degenerate electron gas," Phys. Kondens. Mater. 6, 193-205 (1967).

[2] L. Hedin and S. Lundqvist, "Effects of electron-electron and electron-phonon interactions on the one-electron states of solids," in Solid State Physics, Vol. 23, edited by F. Seitz, D. Turnbull, and H. Ehrenreich (Academic Press, New York, 1969) pp. 1-181.

[3] D. C. Langreth, "Singularities in the X-ray spectra of metals," Phys. Rev. B 1, 471-477 (1970).

[4] Y. Baer and G. Busch, "X-ray photoemission from aluminum," Phys. Rev. Lett. 30, 280-282 (1973).

[5] A. Bostwick, F. Speck, T. Seyller, K. Horn, M. Polini, R. Asgari, A. H. MacDonald, and E. Rotenberg, "Observation of plasmarons in quasi-freestanding doped graphene," Science 328, 999-1002 (2010).

[6] S. Moser, L. Moreschini, J. Jaćimović, O. S. Barišić, H. Berger, A. Magrez, Y. J. Chang, K. S. Kim, A. Bostwick, E. Rotenberg, L. Forró, and M. Grioni, "Tunable polaronic conduction in anatase $\mathrm{TiO}_{2}$," Phys. Rev. Lett. 110, 196403 (2013).

[7] Z. Wang, S. McKeown Walker, A. Tamai, Y. Wang, Z. Ristic, F. Y. Bruno, A. de la Torre, S. Riccò, N. C. Plumb, M. Shi, P. Hlawenka, J. Sánchez-Barriga, A. Varykhalov, T. K. Kim, M. Hoesch, P. D. C. King, W. Meevasana, U. Diebold, J. Mesot, B. Moritz, T. P. Devereaux, M. Radovic, and F. Baumberger, "Tailoring the nature and strength of electron-phonon interactions in the $\mathrm{SrTiO}_{3}(001)$ two-dimensional electron liq- uid," Nat. Mater. 15, 835-839 (2016).

[8] A. Lanzara, P. V. Bogdanov, X. J. Zhou, S. A. Kellar, D. L. Feng, E. D. Lu, T. Yoshida, H. Eisaki, A. Fujimori, K. Kishio, J.-I. Shimoyama, T. Noda, S. Uchida, Z. Hussain, and Z.-X. Shen, "Evidence for ubiquitous strong electron-phonon coupling in high-temperature superconductors," Nature 412, 510 (2001).

[9] A. Damascelli, Z. Hussain, and Z.-X. Shen, "Angleresolved photoemission studies of the cuprate superconductors," Rev. Mod. Phys. 75, 473 (2003).

[10] S. Logothetidis, J. Petalas, H. M. Polatoglou, and D. Fuchs, "Origin and temperature dependence of the first direct gap of diamond," Phys. Rev. B 46, 4483-4494 (1992).

[11] F. Giustino, S. G. Louie, and M. L. Cohen, "Electronphonon renormalization of the direct band gap of diamond," Phys. Rev. Lett. 105, 265501 (2010).

[12] S. Poncé, Y. Gillet, J. Laflamme Janssen, A. Marini, M. Verstraete, and X. Gonze, "Temperature dependence of the electronic structure of semiconductors and insulators," J. Chem. Phys. 143, 102813 (2015).

[13] A. Eiguren, B. Hellsing, F. Reinert, G. Nicolay, E. V. Chulkov, V. M. Silkin, S. Hüfner, and P. M. Echenique, "Role of bulk and surface phonons in the decay of metal surface states," Phys. Rev. Lett. 88, 066805 (2002).

[14] C.-H. Park, F. Giustino, M. L. Cohen, and S. G. Louie, "Velocity renormalization and carrier lifetime in graphene from the electron-phonon interaction," Phys. Rev. Lett. 99, 086804 (2007). 
[15] G.D. Mahan, Many-Particle Physics (Springer, New York, 2000).

[16] P. B. Allen and V. Heine, "Theory of the temperature dependence of electronic band structures," J. Phys. C 9, 2305 (1976).

[17] F. Giustino, "Electron-phonon interactions from first principles," Rev. Mod. Phys. 89, 015003 (2017).

[18] F. Caruso, H. Lambert, and F. Giustino, "Band structures of plasmonic polarons," Phys. Rev. Lett. 114, 146404 (2015).

[19] F. Caruso and F. Giustino, "Spectral fingerprints of electron-plasmon coupling," Phys. Rev. B 92, 045123 (2015).

[20] J. Lischner, G. K. Pálsson, D. Vigil-Fowler, S. Nemsak, J. Avila, M. C. Asensio, C. S. Fadley, and S. G. Louie, "Satellite band structure in silicon caused by electronplasmon coupling," Phys. Rev. B 91, 205113 (2015).

[21] B. Gumhalter, V. Kovač, F. Caruso, H. Lambert, and F. Giustino, "On the combined use of $G W$ approximation and cumulant expansion in the calculations of quasiparticle spectra: The paradigm of Si valence bands," Phys. Rev. B 94, 035103 (2016).

[22] F. Caruso, C. Verdi, S. Poncé, and F. Giustino, "Electron-plasmon and electron-phonon satellites in the angle-resolved photoelectron spectra of $n$-doped anatase $\mathrm{TiO}_{2}$," Phys. Rev. B 97, 165113 (2018).

[23] C. Verdi, F. Caruso, and F. Giustino, "Origin of the crossover from polarons to Fermi liquids in transition metal oxides," Nat. Commun. 8, 15769 (2017).

[24] W. G. Aulbur, L. Jonsson, and J. W. Wilkins, "Quasiparticle calculations in solids," in Solid State Physics, Vol. 54, edited by Henry Ehrenreich and Frans Spaepen (Academic Press, 2000) pp. 1-218.

[25] W. Kohn and L. J. Sham, "Self-consistent equations including exchange and correlation effects," Phys. Rev. 140, A1133-A1138 (1965)

[26] A. Marini, S. Poncé, and X. Gonze, "Many-body perturbation theory approach to the electron-phonon interaction with density-functional theory as a starting point," Phys. Rev. B 91, 224310 (2015).

[27] A. Marini, C. Hogan, M. Grüning, and D. Varsano, "Yambo: An ab initio tool for excited state calculations," Comput. Phys. Commun. 180, 1392-1403 (2009).

[28] S. Poncé, E. R. Margine, C. Verdi, and F. Giustino, "EPW: Electron-phonon coupling, transport and superconducting properties using maximally localized Wannier functions," Comput. Phys. Commun. 209, 116-133 (2016).

[29] J. J. Kas, J. J. Rehr, and L. Reining, "Cumulant expansion of the retarded one-electron Green function," Phys. Rev. B 90, 085112 (2014).

[30] F. Aryasetiawan, L. Hedin, and K. Karlsson, "Multiple plasmon satellites in $\mathrm{Na}$ and $\mathrm{Al}$ spectral functions from $a b$ initio cumulant expansion," Phys. Rev. Lett. 77, 22682271 (1996).

[31] F. Aryasetiawan and O. Gunnarson, "The $G W$ method," Rep. Prog. Phys. 61, 237 (1998).

[32] L. Hedin, "Effects of recoil on shake-up spectra in metals," Phys. Scr. 21, 477 (1980).

[33] J. S. Zhou, J. J. Kas, L. Sponza, I. Reshetnyak, M. Guzzo, C. Giorgetti, M. Gatti, F. Sottile, J. J. Rehr, and L. Reining, "Dynamical effects in electron spectroscopy," J. Chem. Phys. 143, 184109 (2015).

[34] B. Holm and F. Aryasetiawan, "Self-consistent cumulant expansion for the electron gas," Phys. Rev. B 56, 1282512831 (1997).

[35] F. Caruso, P. Rinke, X. Ren, A. Rubio, and M. Scheffler, "Self-consistent $G W$ : All-electron implementation with localized basis functions," Phys. Rev. B 88, 075105 (2013).

[36] F. Caruso, M. Dauth, M. J. van Setten, and P. Rinke, "Benchmark of $G W$ approaches for the GW100 test set," J. Chem. Theory Comput. 12, 5076-5087 (2016).

[37] A. Kutepov, K. Haule, S. Y. Savrasov, and G. Kotliar, "Electronic structure of $\mathrm{Pu}$ and Am metals by selfconsistent relativistic $G W$ method," Phys. Rev. B 85, 155129 (2012).

[38] B. Holm and U. von Barth, "Fully self-consistent $G W$ self-energy of the electron gas," Phys. Rev. B 57, 2108 (1998).

[39] F. Caruso and F. Giustino, "Theory of electron-plasmon coupling in semiconductors," Phys. Rev. B 94, 115208 (2016).

[40] H. Fröhlich, "Electrons in lattice fields," Adv. Phys. 3, 325 (1954).

[41] C. Verdi and F. Giustino, "Fröhlich electron-phonon vertex from first principles," Phys. Rev. Lett. 115, 176401 (2015).

[42] J. Sjakste, N. Vast, M. Calandra, and F. Mauri, "Wannier interpolation of the electron-phonon matrix elements in polar semiconductors: Polar-optical coupling in GaAs," Phys. Rev. B 92, 054307 (2015).

[43] F. Giustino, M. L. Cohen, and S. G. Louie, "Electronphonon interaction using Wannier functions," Phys. Rev. B 76, 165108 (2007).

[44] S. M. Story, J. J. Kas, F. D. Vila, M. J. Verstraete, and J. J. Rehr, "Cumulant expansion for phonon contributions to the electron spectral function," Phys. Rev. B 90, 195135 (2014).

[45] P. Steiner, H. Höchst, and S. Hüfner, "Photoemission in solids II," in Topics in Applied Physics Vol. 27, edited by L. Ley and M. Cardona (Springer-Verlag, Heidelberg, 1979, Heidelberg, 1979).

[46] M. Guzzo, G. Lani, F. Sottile, P. Romaniello, M. Gatti, J. J. Kas, J. J. Rehr, M. G. Silly, F. Sirotti, and L. Reining, "Valence electron photoemission spectrum of semiconductors: Ab initio description of multiple satellites," Phys. Rev. Lett. 107, 166401 (2011).

[47] S. Hüfner, Photoelectron Spectroscopy, 3rd ed. (Springer, Berlin, 2003).

[48] M. Guzzo, J. J. Kas, F. Sottile, M. G. Silly, F. Sirotti, J. J. Rehr, and L. Reining, "Plasmon satellites in valence-band photoemission spectroscopy," Eur. Phys. J. B 85, 324 (2012).

[49] Y. J. Chang, A. Bostwick, Y. S. Kim, K. Horn, and E. Rotenberg, "Structure and correlation effects in semiconducting $\mathrm{SrTiO}_{3}$," Phys. Rev. B 81, 235109 (2010).

[50] J. J. Lee, F. T. Schmitt, R. G. Moore, S. Johnston, Y.-T. Cui, W. Li, M. Yi, Z. K. Liu, M. Hashimoto, Y. Zhang, D. H. Lu, T. P. Devereaux, D.-H. Lee, and Z.-X. Shen, "Interfacial mode coupling as the origin of the enhancement of $T_{\mathrm{c}}$ in FeSe films on $\mathrm{SrTiO}_{3}$," Nature 515, 245248 (2014).

[51] P. D. C. King, S. Mckeown Walker, A. Tamai, A. de la Torre, T. Eknapakul, P. Buaphet, S.-K. Mo, W. Meevasana, M. S. Bahramy, and F. Baumberger, "Quasiparticle dynamics and spin-orbital texture of the $\mathrm{SrTiO}_{3}$ two-dimensional electron gas," Nat. Commun 5, 
3414 (2014).

[52] C. Chen, J. Avila, E. Frantzeskakis, A. Levy, and M. C Asensio, "Observation of a two-dimensional liquid of Fröhlich polarons at the bare $\mathrm{SrTiO}_{3}$ surface," Nat. Commun. 6, 8585 (2015).

[53] C. Cancellieri, A. S. Mishchenko, U. Aschauer, A. Filippetti, C. Faber, O. S. Barišić, V. A. Rogalev, T. Schmitt, N. Nagaosa, and V. N. Strocov, "Polaronic metal state at the $\mathrm{LaAlO}_{3} / \mathrm{SrTiO}_{3}$ interface," Nat. Commun 7, 10386 (2016).

[54] R. Yukawa, K. Ozawa, S. Yamamoto, H. Iwasawa, K. Shimada, E. F. Schwier, K. Yoshimatsu, H. Kumigashira, H. Namatame, M. Taniguchi, and I. Matsuda, "Phonondressed two-dimensional carriers on the $\mathrm{ZnO}$ surface." Phys. Rev. B 94, 165313 (2016).

[55] L. Rademaker, Y. Wang, T. Berlijn, and S. Johnston, "Enhanced superconductivity due to forward scattering in FeSe thin films on $\mathrm{SrTiO}_{3}$ substrates," New J. Phys. 18, 022001 (2016).

[56] G. Antonius, S. Poncé, É Lantagne-Hurtubise, G. Auclair, X. Gonze, and M. Côté, "Dynamical and anharmonic effects on the electron-phonon coupling and the zero-point renormalization of the electronic structure," Phys. Rev. B 92, 085137 (2015).

[57] J. P. Nery, P. B. Allen, G. Antonius, L. Reining,
A. Miglio, and X. Gonze, "Quasiparticles and phonon satellites in spectral functions of semiconductors and insulators: Cumulants applied to the full first principles theory and the Fröhlich polaron," Phys. Rev. B 97, 115145 (2018).

[58] C. Kittel, Introduction to Solid State Physics, 5th ed. (Wiley, New York, 1976).

[59] L. Hedin, "New method for calculating the one-particle Green's function with application to the electron-gas problem," Phys. Rev. 139, A796 (1965).

[60] J. M. Riley, F. Caruso, C. Verdi, L. B. Duffy, M. D. Watson, L. Bawden, K. Volckaert, G. van der Laan, T. Hesjedal, M. Hoesch, F. Giustino, and P. D. C. King, "Crossover from lattice to plasmonic polarons of a spinpolarised electron gas in ferromagnetic EuO," Nat. Commun. 9, 2305 (2018).

[61] B. B. Varga, "Coupling of plasmons to polar phonons in degenerate semiconductors," Phys. Rev. 137, A1896 (1965).

[62] M. Settnes, J. R. M. Saavedra, K. S. Thygesen, A.-P. Jauho, F. J. García de Abajo, and N. A. Mortensen, "Strong plasmon-phonon splitting and hybridization in 2D materials revealed through a self-energy approach," ACS Photon. 4, 2908 (2017). 